\title{
RADIOLOGICAL ASPECTS OF PULMONARY ATRESIA WITH INTACT VENTRICULAR SEPTUM*
}

\author{
BY \\ STEPHEN A. KIEFFER AND LEWIS S. CAREY \\ From the Department of Radiology, University of Minnesota Hospitals, Minneapolis, Minnesota, U.S.A.
}

Received January 7, 1963

The clinical, pathological, and electrocardiographic findings in congenital pulmonary atresia with intact septum have been reviewed recently by Elliott, Adams, and Edwards (1963). Among their 12 cases, the x-ray examinations on 10 were available for this study.

The most characteristic appearance on the frontal chest radiograph in pulmonary atresia is the diminution in the size of the pulmonary vasculature. All 10 cases in this series showed moderate or great decrease in the pulmonary vasculature. Admittedly, the newborn child caught in the extreme depth of inspiration on a good cry may give the appearance of having decreased pulmonary vasculature but not to the degree seen in any of these children.

Associated with the decreased pulmonary vasculature one usually finds a degree of cardiomegaly. It is noteworthy that most of these 10 infants did not exhibit much cardiomegaly. The only case in which cardiomegaly was pronounced was that of an infant who, at necropsy, was found to have a large right ventricular cavity.

The configuration of the heart in most cases of pulmonary atresia is fairly typical (Fig. 1). The right cardiac border may show two convex curves. Superiorly one may note a prominent convex soft tissue density caused by an enlarged prominent aorta which displaces the superior vena cava to the right. The inferior convex density is the result of an enlarged right atrium. The superior portion of the left cardiac border in the region of the pulmonary artery segment is usually concave, which reflects the absence or underdevelopment of the main pulmonary artery. This region was concave, or at best flattened, in all 10 cases. The lower left cardiac border, however, shows a prominent convexity which appears to point more laterally than inferiorly: this prominence can be attributed to the enlarged left ventricular chamber. Furthermore, oblique and lateral views of the heart usually show evidence of left atrial enlargement.

\section{ANGIOCARDIOGRAPHY}

Forward angiocardiograms were available for review in 7 of the 10 cases. All the angiocardiographic studies had been performed through the saphenous route.

The inferior vena cava was well shown in each case and appeared normal in calibre. Reflux of contrast media from the right atrium into the superior vena cava occurred in three instances and showed lateral displacement by an enlarged aorta (Fig. 2).

All cases showed enlargement of the right atrium. Contrast medium injected through the saphenous vein opacifies the inferior vena cava and appears to flow into the right atrial cavity in a straight

* This study was aided by National Heart Institute Grants HE-5694 and HTS-5222. United States Public Health Service. 


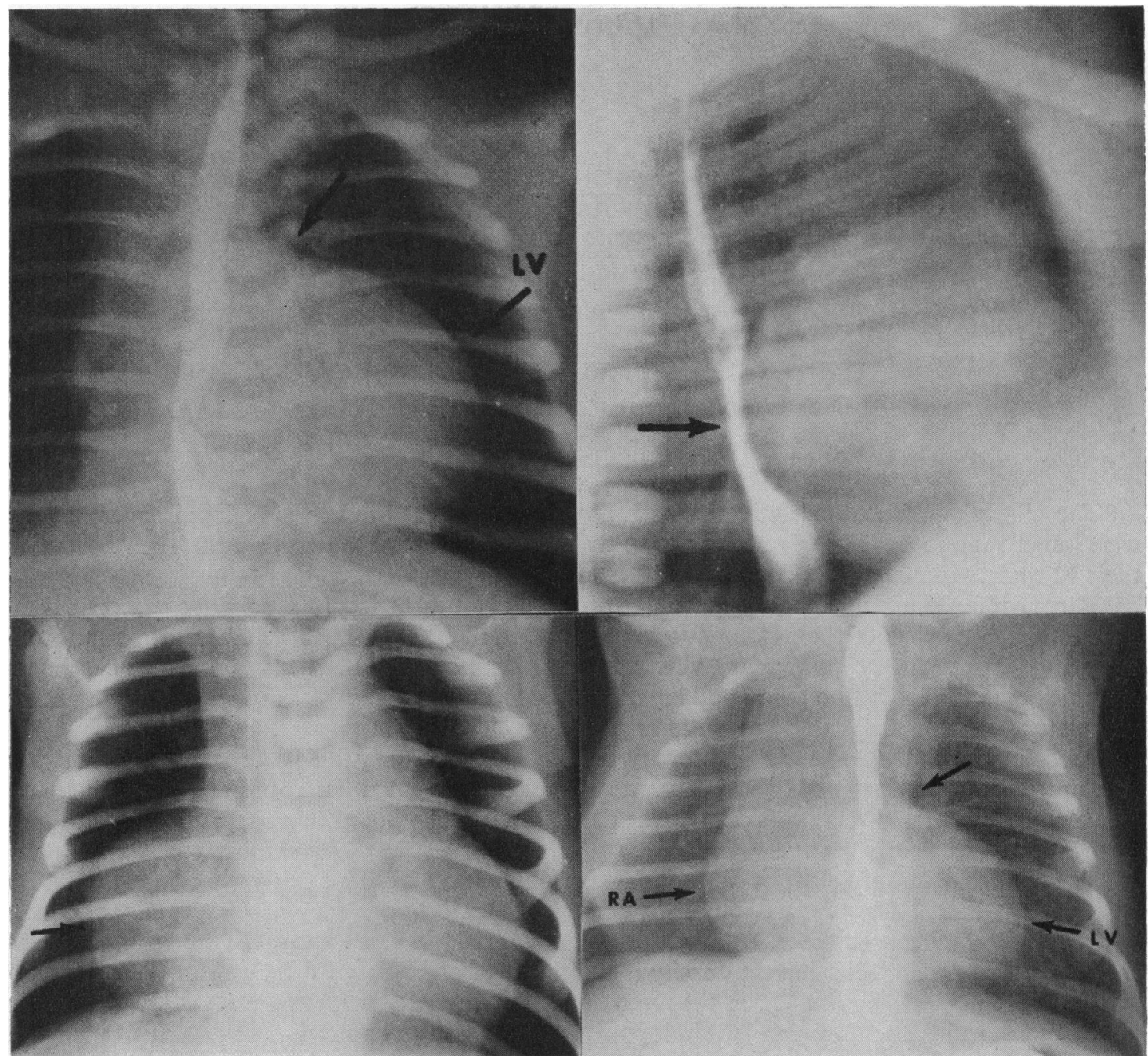

FIG. 1.-Salient features in the conventional radiograph. Upper left: Frontal view demonstrates prominence of the left ventricle (LV) and the concavity of superior portion of left border as a result of the underdevelopment of the outflow portion of the right ventricle and main pulmonary artery (arrow). Upper right: Lateral view shows enlargement of the left atrium (arrow). The prominence of the anterior and superior aspect of the cardiac density is the result of an enlarged right atrial appendage. Lower left: Frontal view in patient with aneurysmal right atrium (arrow). Lower right: Photo shows the prominence of the right atrium (RA) on the right border and of the left ventricle which comprises the major portion of the left heart border. The third arrow points to the concavity in the region of the pulmonary artery segment.

line. The column of contrast medium then appears to curve posteriorly through the atrial septal defect (Fig. 3A). When the contrast medium is passing from the inferior vena cava into the heart, it is often noted that the right atrial chamber fills only faintly, probably because the main bolus is directed by streaming through the atrial septal defect. Ordinarily, it is only after the left atrial cavity is opacified that contrast medium spreads throughout the confines of the dilated right atrial chamber (Fig. 3B).

The left atrium is also enlarged but not to the extent of the right atrium. The lateral films are particularly helpful in visualizing the left atrium, which lies behind and slightly cephalad to the pos- 


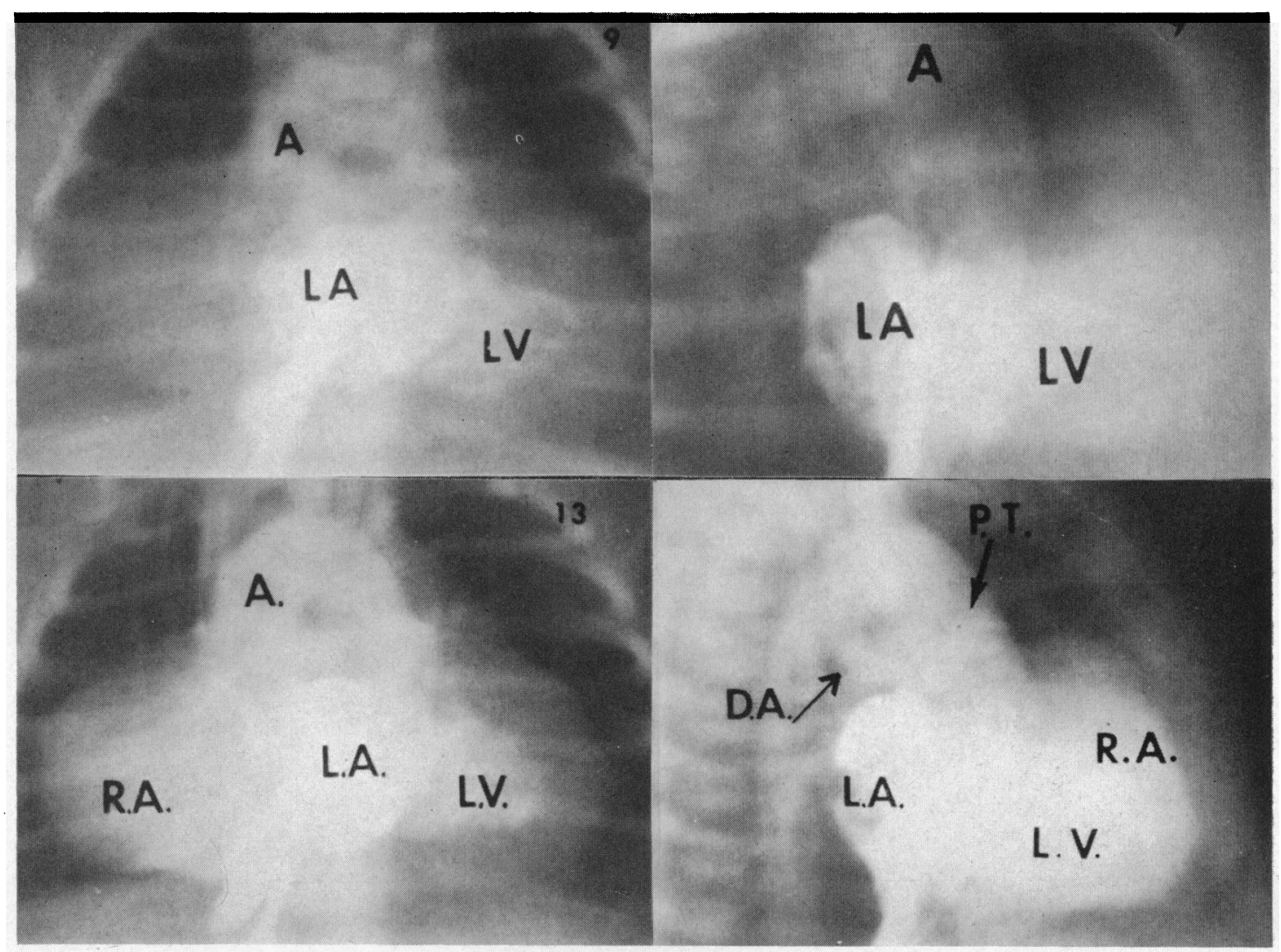

Fig. 2.-Biplane forward angiocardiogram. Upper left and right. Films made at the rate of five per second. The number on each film indicates its position in the film study. Films show direct streaming of contrast medium from inferior vena cava into left atrium (LA). Contrast media also faintly outlines the left ventricle (LV) and aorta (A). Lower left. Later films show diffuse opacification of right atrium with accumulation of contrast chiefly in left atrium. Left ventricle and aorta are now seen to fill more densely. The superior vena cava is displaced laterally by the enlarged aorta. The striking hypertrophy of the wall of left ventricle is well shown. Lower right. Lateral view shows filling of the pulmonary trunk (PT) as a linear density overlying the aorta. Filling is through the ductus arteriosus (DA).

terior wall of the right atrial chamber. On the lateral film, it is common to note the streaming of contrast medium from the right atrium through the atrial septal defect into the antero-superior portion of the left atrium (Fig. 3A). The main bolus then appears to course around the wall of the left atrium, describing the arc of a circle, until it reaches the mitral valve. The left atrial appendage also shares in this enlargement, and this structure was prominent and filled with contrast in five of these seven cases.

The left atrium may become quite densely opacified before the contrast fills the left ventricle. The left ventricle is enlarged and its wall is usually well defined by the contrast medium. The hypertrophy of the musculature of the left ventricle is usually striking in degree (Fig. 4).

At this point in the study, the cardiac density is almost completely filled with contrast medium: the enlarged right atrium is faintly, but definitely, outlined; the left atrium is filled quite densely; and the left ventricle has now accumulated enough contrast to outline its walls.

Invariably the aorta is enlarged. The root of the aorta lies in its normal posterior position as visualized on the lateral films. Following opacification of the arch of the aorta, a patent ductus arteriosus was noted in all cases and, immediately subsequent to this event, the pulmonary 


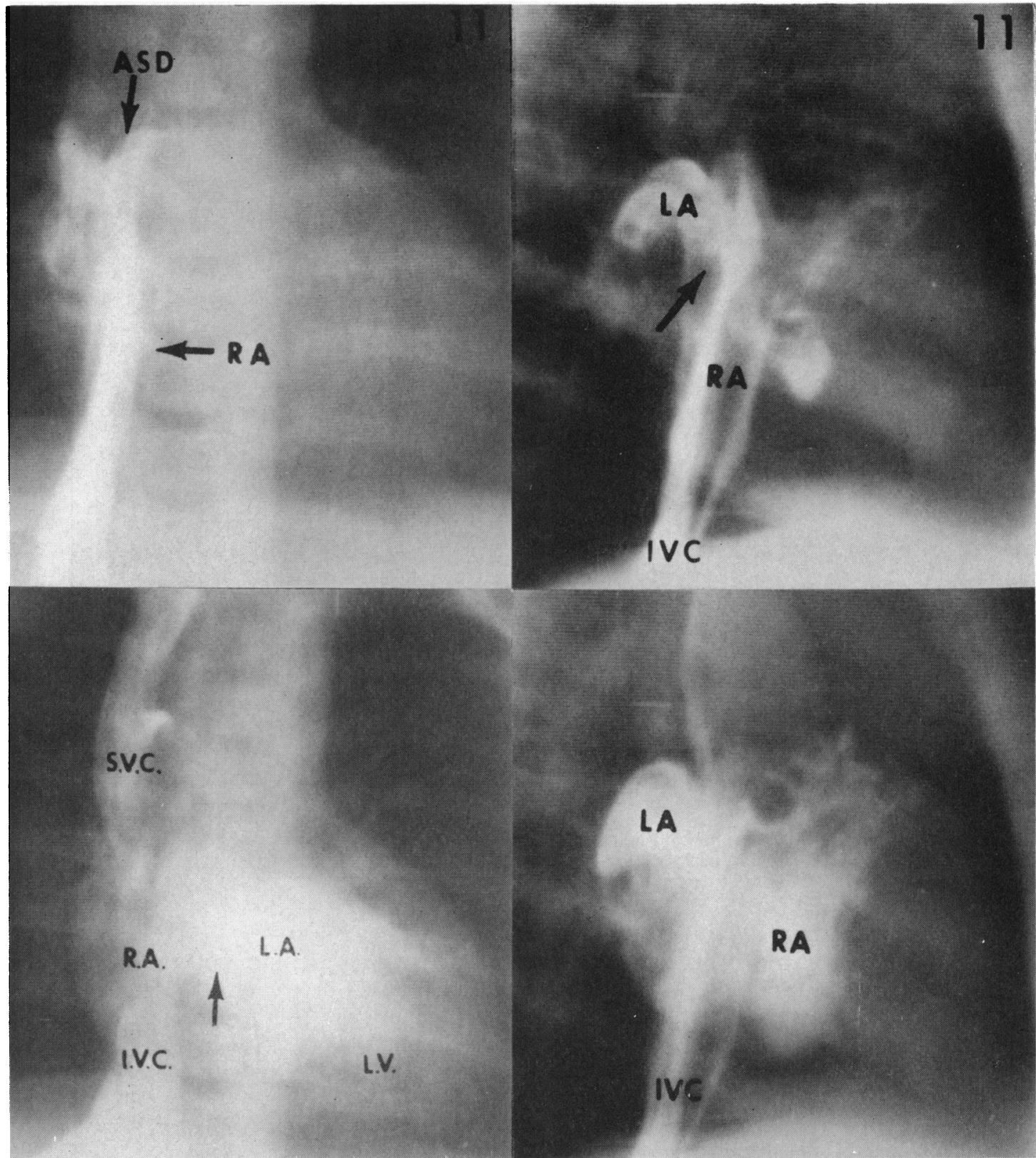

A

FIG. 3.-Biplane forward angiocardiogram. Films made at rate of five per second. The number on each film indicates its position in the film study. A. Upper left and right. Frontal and lateral views. Films demonstrate the rapid streaming of contrast bolus through the right atrium (RA) and the atrial septal defect (ASD) into the left atrium. The unlabelled arrow on the lateral film points to the inferior aspect of the atrial septal defect. Lower left and right: Films show reflux from the right atrium into the superior vena cava and azygos vein. Arrow points to apex of "right ventricular notch" which actually represents right atrium not yet filled with contrast medium. $\mathrm{LA}=$ left atrium; $\mathrm{RA}=$ right atrium; $\mathrm{IVC}=$ inferior vena cava. 


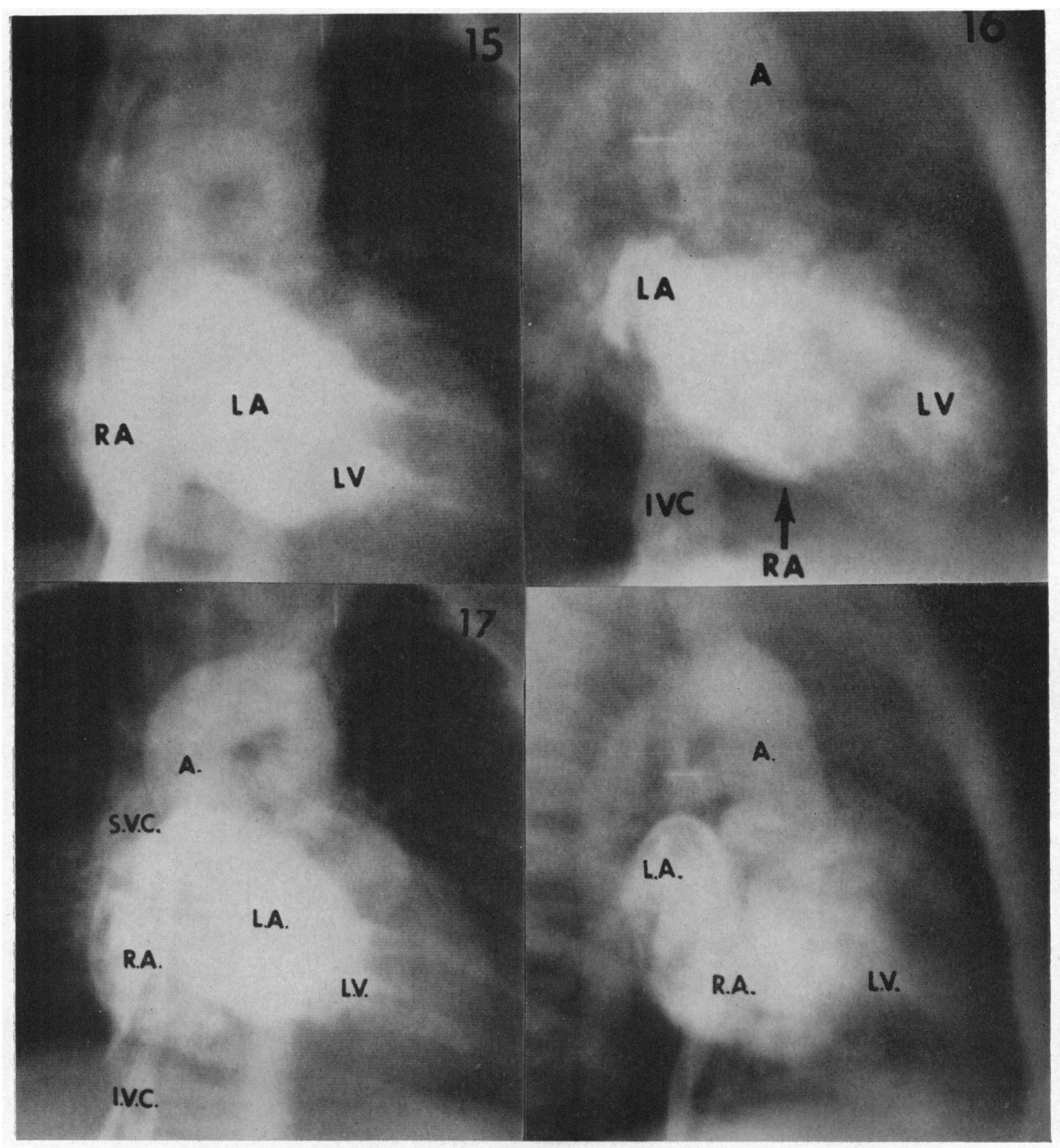

B

FIG. 3.-B. Upper left. Frontal view. Contrast material is seen in the left ventricle and also faintly outlines the aorta. Right atrium is now better filled with contrast, and the "right ventricular notch" is less apparent. Right. Lateral view. Note the area of unopacified heart density superior to the left ventricle (LV). This is the location of the small right ventricular cavity. Lower left: Frontal view. All chambers of the heart except the right ventricle now are well delineated by contrast medium. Note especially the enlarged left atrium (LA). Right. Lateral view shows filling of the pulmonary trunk via the ductus arteriosus. 


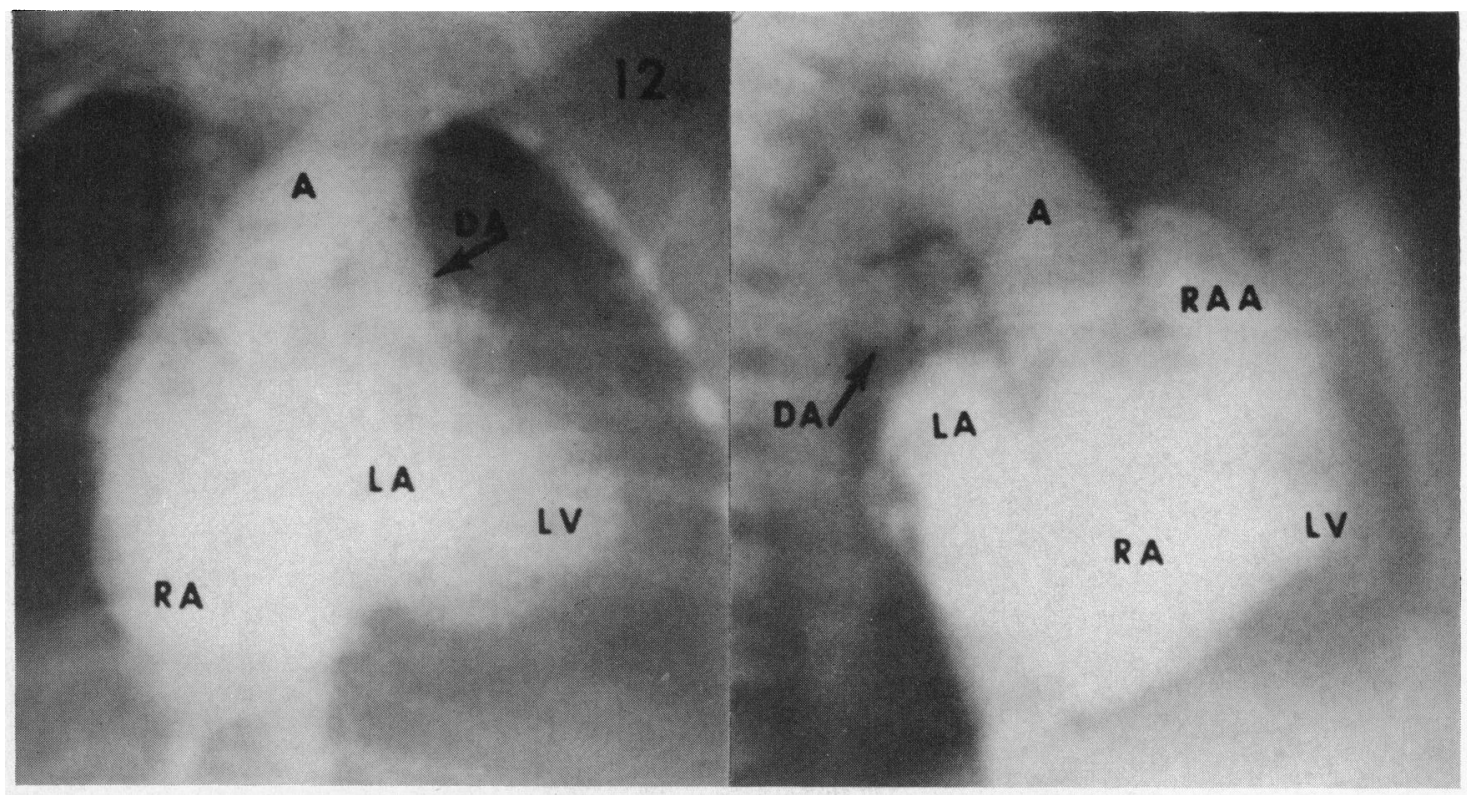

FIG. 4.-Left and right. Biplane forward angiocardiogram. Frontal and lateral views. Late films show diffuse filling of the right atrium. Faint filling of the large patent ductus arteriosus (DA) is also noted. Lateral film demonstrates the location of the enlarged right atrial appendage (RAA). This overlies the area of the small right ventricular chamber. Marked hypertrophy of the wall of the left ventricle is shown.

vasculature filled via the ductus (Fig. 3B and 4). Left and right main pulmonary arteries usually appear smaller than normal and the peripheral pulmonary vessels are often no larger than small threads. The entire pulmonary trunk may become opacified via the ductus down to the level of the atretic valve and, in this manner, the small fused dome of the atretic valve may be seen.

\section{Discussion}

In view of the surgical implications, once the diagnosis of pulmonary atresia with intact ventricular septum has been made, the most important question to answer is: How large is the right ventricular chamber? Anatomically, it has been proved that the right ventricular chamber is present and that it varies in size. The lack of thrombus material in the right ventricle at necropsy suggests some circulation of blood into this chamber via the hypoplastic tricuspid valve.

In 1960, Paul and Lev described a cineangiographic study of the circulation in the small right ventricle of a patient having pulmonary atresia with intact ventricular septum. Contrast material injected rapidly through a catheter whose tip was placed in the right ventricular cavity remained in that cavity for a period of 10 seconds. During this interval, these authors noted a gradual dilution of the contrast medium, along with evidence for regurgitation through the tricuspid valve. They concluded that this slow bidirectional flow of blood prevented the obliteration of the right ventricular cavity by stasis and thrombosis.

On none of the films from our seven forward angiocardiographic studies were we able definitely to identify contrast media in a right ventricular cavity. It may be that contrast material within this cavity is obscured by overlying medium in the enlarged right atrium. It is also possible that the streaming effect of the material allows inadequate visualization of the right ventricular chamber.

That the small right ventricle can be visualized with contrast medium has been proved by Keith, Rowe, and Vlad (1958) and Davignon et al. (1961). The latter investigators have reported selective 
angiocardiographic examinations in two cases of pulmonary atresia with intact ventricular septum. In each case, films demonstrated the size of the right ventricle after injection of contrast material through a catheter with its tip in that chamber. It is apparent from our experience that forward angiocardiography will not demonstrate the location or size of the right ventricle with accuracy. Because of the importance of making this evaluation for the surgeon attempting to perform pulmonary valvotomy, we think that, whenever possible, a catheter should be introduced into the right side of the heart and an attempt made to gain entrance into the right ventricle so that contrast may be injected at this site. Failing this, a selective study performed with the tip of the catheter in the right atrial chamber should be made.

On none of the seven forward angiocardiograms were we able to delineate the anomalous coronary sinusoids and vessels described by Elliott et al. (1963).

The presence of a "notch" sign on the frontal projection in pulmonary atresia and also in tricuspid atresia has been cited previously as evidence for the presence of a right ventricular chamber. The "notch" refers to a portion of unopacified cardiac density which overlies the thoracic spine and extends upwards from the diaphragmatic border of the heart (Fig. 3A). The size of this area has been related to the size of the right ventricular cavity. However, we believe that the "notch" represents only an unfilled area of right atrium in an early stage of the progression of contrast medium as it streams from the right atrium into the left atrium. The apex of the "notch" is formed by the contrast medium passing through the atrial septal defect. In one case we are able to identify an area free of contrast media antero-superiorly. This is the anatomical location of the small right ventricle in pulmonary atresia with intact ventricular septum (Fig. 3B).

Differential Diagnosis. The two chief entities to be considered in the differential diagnosis of pulmonary atresia with intact ventricular septum are pseudotruncus and tricuspid atresia. Differentiation between pulmonary atresia with intact septum and pulmonary atresia with ventricular septal defect (pseudotruncus) is usually not difficult. In the former instance, it will be noted that the aorta fills from the left ventricle: in the latter, an enlarged right ventricular cavity can usually be identified, and it may be noted that the aorta fills readily with contrast medium from the right ventricular chamber via the ventricular septal defect.

Differentiation between pulmonary atresia with intact septum and small right ventricle on the one hand and tricuspid atresia on the other is difficult by forward angiocardiography since in both instances the hæmodynamics are similar. The electrocardiogram has been helpful in differentiating these two types, and sometimes in predicting the size of the right ventricular chamber in cases of pulmonary atresia. One feature that may be helpful in separating these two types of malformation has to do with the size of the inferior vena cava. Castellanos, García, and González (1960) have recently described a "mega vena cava" sign in tricuspid atresia. Some, but not all, of these cases show a noteworthy widening of the inferior vena cava possibly due to a raised pressure in the right atrium. The inferior vena cava in all our cases of pulmonary atresia was well within normal limits as to size. The significance of this finding is not clear since both conditions have a raised right atrial pressure. We have also observed a large inferior vena cava in tricuspid atresia. Such a finding is not unique for this malformation, since it has also been seen in other types of congenital heart disease. One other differential point of value concerns the manner of opacification of the great vessels. Invariably in our experience in pulmonary atresia the pulmonary trunk opacifies via a patent ductus following opacification of the aortic arch. In contrast, in some cases of tricuspid atresia, because of the associated ventricular septal defect, simultaneous opacification of both great vessels from the left ventricle may be noted.

\section{SUMMARY}

The radiological findings on the chest of 10 children observed at the University of Minnesota Hospitals and proved at necropsy to have congenital pulmonary atresia with intact ventricular septum have been reviewed. In addition, angiocardiographic studies were available on seven of 
these cases, and these have been evaluated. Forward angiocardiography is a valuable procedure in the diagnosis of this lesion, but selective injection into the right ventricle may be necessary to delineate the size of that chamber before attempting corrective surgery.

\section{REFERENCES}

Castellanos, A., García, O., and González, E. (1960). Atresia tricuspidia en la infancia. Su diagnóstico angiocardiográfico. Radiologia (Panamá), 11, 21.

Davignon, A. L., DuShane, J. W., Kincaid, O. W., and Swan, H. J. C. (1961). Pulmonary atresia with intact ventricular septum. Report of two cases studied by selective angiocardiography and right heart catheterization. Amer. Heart J., 62, 690.

Elliott, L. P., Adams, P., and Edwards, J. E. (1963). Pulmonary atresia with intact ventricular septum. Anatomical, electrocardiographic, and clinical observations. Brit. Heart J., 25, 489.

Keith, J. D., Rowe, R. D., and Vlad, P. (1958). Heart Disease in Infancy and Childhood. Macmillan, New York.

Paul, M. H., and Lev, M. (1960). Tricuspid stenosis with pulmonary atresia. A cineangiographic-pathologic correlation. Circulation, 22, 198. 